\title{
Spontaneous coronary artery dissections and associated predisposing factors: a narrative review
}

\author{
E. B. N. J. Janssen · P. W. de Leeuw - A. H. E. M. Maas
}

Published online: 25 January 2019

(c) The Author(s) 2019

\begin{abstract}
Spontaneous coronary artery dissection (SCAD) represents around $25 \%$ of cases of acute coronary syndromes (ACS) in women aged 40-65 years who have few or no traditional cardiovascular risk factors. It is assumed that the incidence is underestimated, as the angiographic appearance of SCAD may often mimic atherosclerosis. This review aims to examine SCAD by focusing on the associated predisposing factors and precipitating stressors in this heterogeneous patient population, as well as the best treatment approach and the prognosis. Progressive knowledge has improved our current understanding of SCAD, but more awareness among clinicians is necessary. Recently, two position papers from the European Society of Cardiology (ESC) and the American Heart Association (AHA) have been released, which will be summarised in brief.
\end{abstract}

Keywords Spontaneous coronary artery dissection . Acute coronary syndrome $\cdot$ Myocardial infarction $\cdot$ Fibromuscular dysplasia · Women

\section{Introduction}

Spontaneous coronary artery dissection (SCAD) is an increasingly acknowledged cause of acute coronary syndromes (ACS) in women aged 40-65 years, with

\section{E. B. N. J. Janssen}

Radboud University, Nijmegen, The Netherlands

\section{P. W. de Leeuw}

Department of Internal Medicine, Maastricht University Medical Centre (MUMC+), Maastricht, The Netherlands

\section{A. H. E. M. Maas (西)}

Department of Cardiology, Radboud University Medical Centre, Nijmegen, The Netherlands

Angela.Maas@radboudumc.nl a peak around 53 years of age (Fig. 1; [1]). In most cases no or few traditional cardiovascular risk factors are present, although in one third of patients hypertension is reported [1-3]. It is estimated that up to $25 \%$ of all ACS in this age group are caused by SCAD, although the diagnosis is often missed [2, 4-7]. Overall, it is estimated that SCAD accounts for $1.7-4 \%$ of all ACS and $0.5 \%$ of sudden cardiac deaths $[4,8,9]$. SCAD is far more prevalent than initially thought, and pregnancy-associated SCAD accounts for a maximum of $10 \%$ of cases. In all patient series there is a very strong female predominance of more than $90 \%$, up to $94 \%$ [1, 7, 10-13]. Patients affected by SCAD are heterogeneous with a variety of underlying predisposing factors and precipitating triggers for the event.

\section{Pathophysiology}

SCAD is defined as a spontaneous tear in the coronary arterial wall due to non-atherosclerotic and noniatrogenic causes $[1,7,14,15]$. The underlying pathophysiology of SCAD is multifactorial, related to underlying arteriopathies, inflammation, hormonal factors and mixed connective tissue diseases, whereas the acute event is often preceded by emotional or physical triggers [7]. Two mechanisms of SCAD are proposed (Fig. 2): first, a spontaneous separation of the coronary arterial wall caused by an intimal tear and resulting in medial dissection, haemorrhage and subsequently the formation of a false lumen $[15,16]$; second and less often, haematoma formation in the media causing separation of two arterial layers and, thereby, leading to the formation of a false lumen and dissection of the true lumen $[15,16]$. Coronary blood flow in SCAD is compromised either directly by the intimal tear or indirectly by compression of the medial haematoma on the coronary artery $[7,16]$. Until now it is uncertain whether a single dominant mecha- 


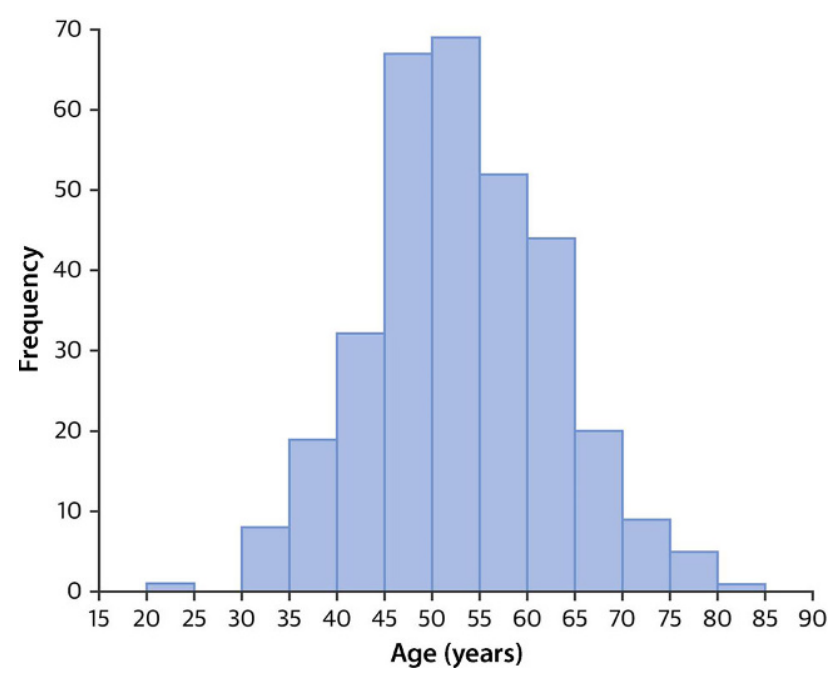

Fig. 1 Age distribution of patients affected by spontaneous coronary artery dissection (SCAD); the mean age of patients with SCAD was $52.5 \pm 9.6$ years, $90.5 \%$ of SCAD patients were 65 years of age or younger. (From Saw et al. [1])

nism causes SCAD or whether both causal events play a role [12]. In more than $50 \%$ of cases the left anterior coronary artery is the affected artery [10, 17].

\section{Triggers for spontaneous coronary artery dissec- tion}

Most cases of SCAD occur in previously healthy young women. Whereas physical stressors are well known, an increasing number of patients report a period of severe emotional distress preceding the acute SCAD event [18]. Research has revealed that severe emotional distress is a potential trigger of an ACS in both women and men [18]. Both emotional and physical stressors create an increase in shear stress which may act as a trigger for dissection [1, 19]. In a study by Saw et al. [1] in 327 SCAD patients, $62 \%$ reported potential precipitating stressors prior their SCAD event, with emotional and physical stressors occurring in $48 \%$ and $28 \%$ of cases, respectively [1].

Emotional precipitating stressors are more common in women than in men $[5,19,20]$. Gender differences in coping with stressful situations may be an important reason why an ACS caused by SCAD is disproportionately predominant in women [21]. Alipour et al. [22] demonstrated that job-related stressors were the most frequently occurring emotional precipitants of SCAD (41\%), followed by death of a loved one $(21 \%)$, arguments $(16 \%)$, relationship breakdown (15\%) and relocation $(10 \%)$. One working hypothesis in SCAD patients is that (prolonged) emotional distress leads to endothelial dysfunction, low thresholds for vascular spams and elevated levels of catecholamines. This may increase the vulnerability for a sudden intimal tear in the vessel wall.

In male patients provoking triggers are more often related to extreme physical exercise, such as competitive mountain biking or weight lifting [19]. Although less common, retching, vomiting, straining with bowel movement and heavy coughing are also reported [19]. Physical precipitating stressors may cause temporary increases in intra-thoracoabdominal pressure leading to a transient rise in coronary arterial wall pressure $[19,23]$.

\section{Predisposing factors}

Although the pathophysiology of SCAD is heterogeneous, it is increasingly acknowledged that several predisposing factors increase the susceptibility for SCAD [12]. Predisposing factors which are associated with SCAD are fibromuscular dysplasia (FMD), female gender, pregnancy-related factors, possibly hormonal therapy, mixed connective tissue disorders and inflammatory disorders [19].

The arteriopathy FMD is an associated disease and the most commonly observed associated factor in these patients $[1,19,23]$. FMD is a non-atherosclerotic, non-inflammatory disease of the musculature of small and medium-sized arteries, leading to stenoses, occlusions, aneurysms or dissections of affected arteries [7, 24, 25]. Like SCAD, FMD has a strong female predominance (>80\%) [26] and commonly affects the renal and carotid arteries [7, 24]. Two dominant types of FMD are nowadays distinguished which angiographically appear as a tubular (unifocal) lesion and as a string-of-beads (multifocal) lesion respectively $[27,28]$. Prior research has found a strong and domi-
Fig. 2 Schematic figure of the two proposed mechanisms of spontaneous coronary artery dissection, a Normal artery, b intraluminal haemorrhage, $\mathbf{c}$ intimal tear. (From Saw et al. [15])
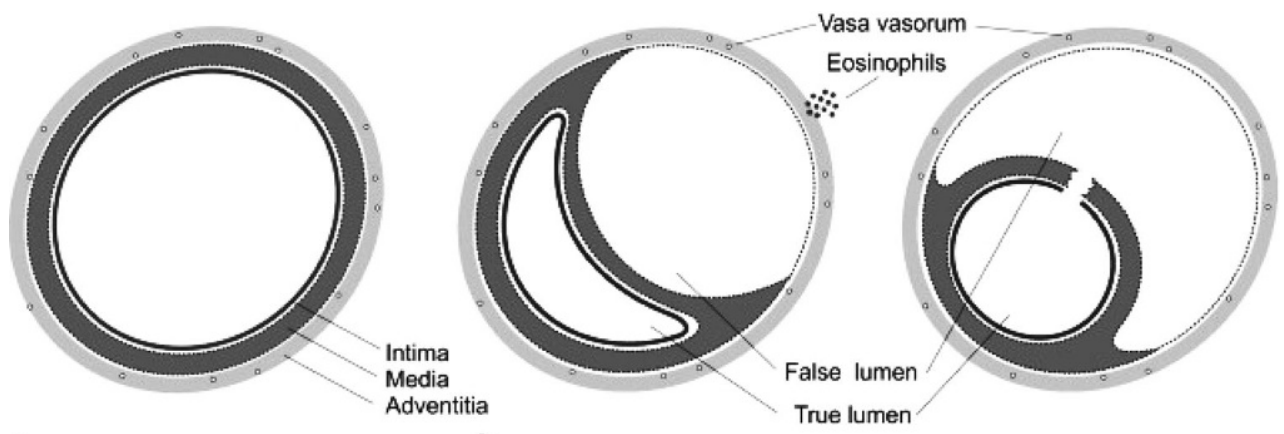

b 
Fig. 3 Angiographic spontaneous coronary artery dissection classification system proposed by Saw et al. [1]

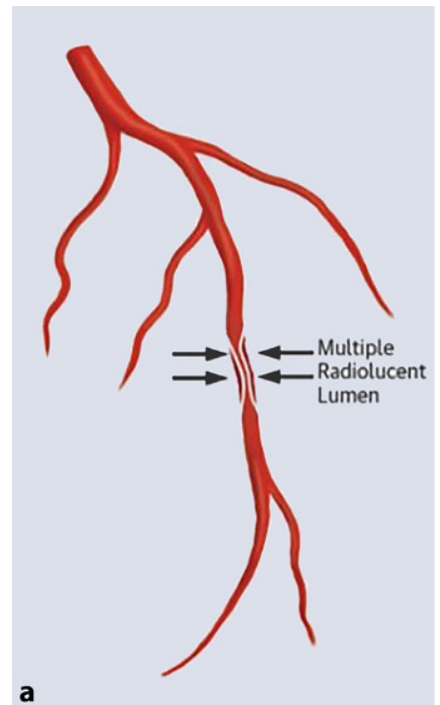

a

nant association of FMD with SCAD with a wide range of $25-86 \%$, which might be due to selection bias or the modality and extent of FMD screening $[1,7,10$, 17, 19, 29-31].

Female gender, pregnancy-related factors and hormonal therapy are also associated with SCAD, suggesting a role for female sex hormones in the pathogenesis of SCAD [12, 17, 32-37]. It is believed that female sex hormones, especially progesterone, which are high in premenopausal women and during pregnancy, influence the integrity of arterial walls [12, 13, 17, 32-37]. However, the precise underlying mechanisms remain unknown.

Although uncommon, mixed connective tissue disorders (MCTD) predispose for SCAD, since these are associated with arterial fragility and vascular dissection $[1,7,13,17,19,23]$. The most common SCADassociated MCTD are Loeys-Dietz syndrome, EhlersDanlos syndrome type 4 and polycystic kidney diseases [1, 7, 23]. Even though only a small proportion of SCAD patients have MCTD $(\leq 5 \%)$, its diagnosis remains important as it may guide monitoring, management and familial screening [11].

Systemic inflammatory disorders associated with SCAD are systemic lupus erythematosus, Crohn's disease, ulcerative colitis, rheumatoid arthritis and coeliac disease [1, 7]. In SCAD infiltrated eosinophils are frequently identified in the adventitia of dissected arteries [38-40]. Whether SCAD is the consequence or the cause of this phenomenon is uncertain.

\section{Clinical diagnosis of spontaneous coronary artery dissection}

A SCAD presents slightly more often as an ST-elevation myocardial infarction (STEMI) than as a nonSTEMI. The clinical picture is consistent with the classical type 1 ACS in most cases. Like any other, the initial diagnosis rests on symptoms, ST-T changes and

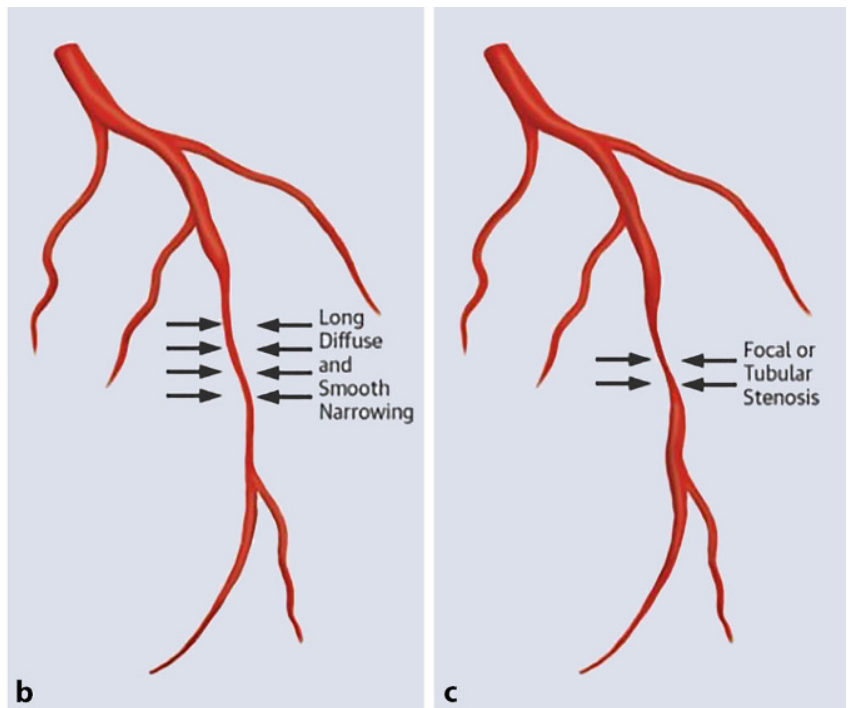

high sensitivity troponin elevations. At coronary angiography, a SCAD may be easily detected. However, typical angiographic SCAD features can be lacking, which is an important reason why SCAD so often goes unrecognised. The false lumen in SCAD is not always clearly visible on angiographic examination and, in addition, SCAD can mimic atherosclerosis [23]. Accurate diagnosis of SCAD is important to provide optimal treatment in the acute phase and thereafter (see next section).

To improve diagnosis, Saw et al. [1] have proposed an angiographic classification in which three main angiographic features of SCAD are presented (Fig. 3). In type 1 the classic appearance of contrast dye staining of the arterial wall with multiple radiolucent lumina is seen [1]. Type 2 appears as a long diffuse and smooth narrowing which varies in severity [1]. This overlaps the 'stick insect' appearance as mentioned in the supplement of the ESC position paper 2018 [12]. Type 3 angiographic SCAD features focal or tubular stenosis and often mimics atherosclerosis [1]. With intracoronary imaging techniques like optical coherence tomography (OCT) and intravascular ultrasound (IVUS) the diagnosis can often be confirmed, but these interventions pose a serious risk for iatrogenic dissection.

\section{Acute and chronic treatment}

Current guidelines for ACS are inappropriate when an ACS is caused by SCAD. As randomised controlled trials for optimal treatment of SCAD are lacking, current recommendations are based on patient series and expert opinions $[12,13]$.

In SCAD patients who are clinically and haemodynamically stable (TIMI score 2 or 3 ) an initial conservative treatment approach is preferred [12, 13, 17, 19, 23, 41-43]. Most (70-97\%) SCAD lesions heal spontaneously within several weeks $[12,13]$. To avoid iatrogenic damage in the already dissected and vul- 
Fig. 4 Algorithm for the management of an acute spontaneous coronary artery dissection. CABG coronary artery bypass graft, $\mathrm{PCl}$ percutaneous coronary intervention. (Adapted from a scientific statement of the American Heart Association [13])

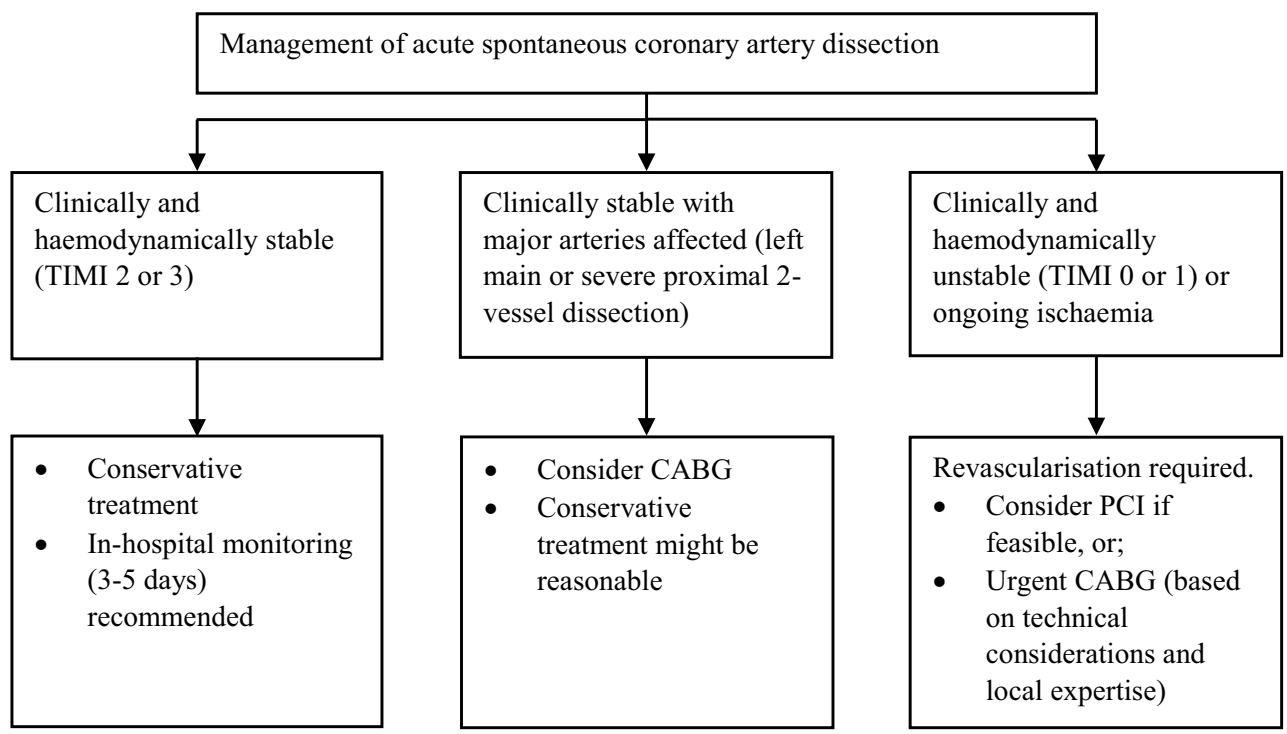

nerable arteries during percutaneous coronary intervention (PCI), this revascularisation technique is not recommended. In experienced hands intracoronary imaging with OCT/IVUS can be performed to establish the correct diagnosis. Although the majority of conservatively treated SCAD patients have an unremarkable in-hospital course, an important minority of approximately $10 \%$ may experience extension of dissection, which may be an indication for acute revascularisation $[12,13,19,41]$. Therefore, prolonged inhospital patient monitoring (3-5 days) after the initial event is recommended in conservatively treated patients $[12,13]$. In SCAD patients who are clinically or haemodynamically unstable (TIMI score 0 or 1 ), who show ongoing ischaemia or in whom major arteries are affected, revascularisation either by PCI or coronary artery bypass graft is required $[1,12,13,17,19$, 42]. Although evidence-based guidelines are lacking, the AHA recently published a treatment algorithm for SCAD treatment (Fig. 4).

As far as follow-up is concerned, the efficacy of standard ACS medical therapy in SCAD patients is still a matter of debate [14]. Only the use of beta-blockers may lower the risk of recurrence, although the 'evidence' is based only on limited retrospective data [1]. In patients with fluctuating residual symptoms the use of diltiazem can be very effective [12]. If patients suffer from hypertension, this should be treated adequately to prevent the recurrence of SCAD. Prolonged use of acetylsalicylic acid after conservative treatment is advised, although the optimal duration of treatment is uncertain. The use of dual antiplatelet therapy should be avoided if no PCI is performed, as this may cause severe menstrual bleeding in premenopausal women. Prolonged use of statins is not advised when lipid levels are normal.

\section{Secondary prevention}

A 'one size fits all' approach is not appropriate in secondary prevention of SCAD. Instead, a more patienttailored prevention program should be offered, including medical management, cardiac rehabilitation, stress management and recommendations regarding physical activity, psychosocial support, contraceptive use and pregnancy if needed [13]. The ESC and AHA recommend SCAD patients to avoid extreme, isometric and competitive physical exercise and to consider psychosocial support [13]. Pregnancy in SCAD survivors requires critical consideration as pregnancy itself is a risk factor and delivery may confer additional physical precipitating distress. Currently, pregnancy is usually not discouraged when the initial event did not cause severe harm and left ventricular function is sufficient. Besides a tailored prevention program, the identification of predisposing factors and precipitating stressors may guide further preventive strategies.

\section{Prognosis}

A recurrent SCAD should be differentiated from extension of a previous dissection and from de novo SCAD. Extension of dissection accounts for $10 \%$ of all recurrent SCAD events and usually occurs within 30 days after the initial event $[41,44]$. A majority of $90 \%$ of recurrent SCAD events is caused by de novo SCAD, usually occurring beyond 30 days after the initial SCAD $[41,44]$. Overall, patient series have reported that recurrent de novo SCAD occurs in $12-27 \%$ of SCAD patients, depending on the duration of follow-up [1]. To date, risk factors for recurrence remain to be further elucidated. Hypertension and coronary tortuosity are presumably associated with a higher recurrence risk $[1,13]$. Whether coronary tortuosity confers a direct risk or appears as a coronary manifestation of FMD is currently unclear $[13,45]$. 


\section{Future directions}

Although our understanding of SCAD has increased enormously over past decades, more knowledge is needed regarding predisposing factors, diagnostics and therapy. Besides, the identification of predisposing stressors will help to improve secondary prevention.

The ESC is currently organising a SCAD registry within the European Observational Research Platform (EOSP) [12]. This registry is expected to start in spring 2019. Within the EORP-SCAD registry genetic studies will be conducted as well.

Presumably, the currently most important task in improving cardiovascular care for SCAD patients is to increase awareness and suspicion among clinicians in the field of cardiology, especially for ACS in younger women. This may be a first step forward in improving survival in this growing patient population.

Acknowledgements The design of this review article was conceptualised by A.H.E.M. Maas and E.B.N.J. Janssen. The article was written by E.B.N.J. Janssen and reviewed by P.W. de Leeuw and A.H.E.M. Maas.

Conflict of interest E.B.N.J. Janssen, P.W. de Leeuw and A.H.E.M. Maas declare that they have no competing interests.

Open Access This article is distributed under the terms of the Creative Commons Attribution 4.0 International License (http://creativecommons.org/licenses/by/4.0/), which permits unrestricted use, distribution, and reproduction in any medium, provided you give appropriate credit to the original author(s) and the source, provide a link to the Creative Commons license, and indicate if changes were made.

\section{References}

1. SawJ, Humphries K, AymongE, et al. Spontaneous coronary artery dissection: clinical outcomes and risk of recurrence. JAm Coll Cardiol. 2017;70:1148-58.

2. Saw J. Coronary angiogram classification of spontaneous coronary artery dissection. Catheter Cardiovasc Interv. 2014;84:1115-22.

3. Tweet MS, Hayes SN, Pitta SR, et al. Clinical features, management, and prognosis of spontaneous coronary artery dissection. Circulation. 2012;126:579-88.

4. Rashid HN, Wong DT, Wijesekera H, et al. Incidence and characterisation of spontaneous coronary artery dissection as a cause of acute coronary syndrome-a single-centre Australian experience. Int JCardiol. 2016;202:336-8.

5. Nakashima T, Noguchi T, Haruta S, et al. Prognostic impact of spontaneous coronary artery dissection in young female patients with acute myocardial infarction: a report from the Angina Pectoris-Myocardial Infarction Multicenter Investigators in Japan. Int JCardiol. 2016;207:341-8.

6. Otten AM, Ottervanger JP, Kloosterman A, et al. Treatment assignment in young women with spontaneous coronary artery dissection. IntJ Cardiol. 2014;176:1223-4.

7. Lebrun S, Bond RM. Spontaneous coronary artery dissection (SCAD): The underdiagnosed cardiac condition that plagues women. Trends Cardiovasc Med. 2017. https://doi. org/10.1016/j.tcm.2017.12.004 (ePub ahead of print).
8. Nishiguchi T, Tanaka A, Ozaki Y, et al. Prevalence of spontaneous coronary artery dissection in patients with acute coronary syndrome. Eur Heart J Acute Cardiovasc Care. 2016;5:263-70.

9. Hill SF, Sheppard MN. Non-atherosclerotic coronary artery disease associated with sudden cardiac death. Heart. 2010;96:1119-25.

10. Saw J, Ricci D, Starovoytov A, Fox R, Buller CE. Spontaneous coronary artery dissection: prevalence of predisposing conditions including fibromuscular dysplasia in a tertiary center cohort. Jacc Cardiovasc Interv. 2013;6:44-52.

11. Henkin S, Negrotto SM, Tweet MS, et al. Spontaneous coronary artery dissection andits association with heritable connective tissue disorders. Heart. 2016;102:876-81.

12. Adlam D, Alfonso F, Maas A, Vrints C. European Society of Cardiology, acute cardiovascular care association, SCAD study group: a position paper on spontaneous coronary artery dissection. Eur Heart J. 2018. https://doi.org/10. 1093/eurheartj/ehy080 (ePub ahead of print).

13. Hayes SN, Kim ESH, Saw J, et al. Spontaneous coronary artery dissection: current state of the science: a scientific statement from the American Heart Association. Circulation. 2018;137:e523-2557.

14. Saw J, Mancini GBJ, Humphries KH. Contemporary review on spontaneous coronary artery dissection. J Am Coll Cardiol. 2016;68:297-312.

15. Saw J. Spontaneous coronary artery dissection. Can J Cardiol. 2013;29:1027-33.

16. Tweet MS, Gulati R, Williamson EE, Vrtiska TJ, Hayes SN. Multimodality imaging for spontaneous coronary artery dissection in women. Jacc Cardiovasc Imaging. 2016;9:436-50.

17. Giacoppo D, Capodanno D, Dangas G, Tamburino C. Spontaneous coronary artery dissection. Int J Cardiol. 2014;175:8-20.

18. Edmondson D, Newman JD, Whang W, Davidson KW. Emotional triggers in myocardial infarction: do they matter? Eur Heart J.2013;34:300-6.

19. Saw J, Aymong E, Sedlak T, et al. Spontaneous coronary artery dissection: association with predisposing arteriopathies and precipitating stressors and cardiovascular outcomes. Circ Cardiovasc Interv. 2014;7:645-55.

20. Fahmy P, Prakash R, Starovoytov A, Boone R, Saw J. Predisposing and precipitating factors in men with spontaneous coronary artery dissection. Jacc Cardiovasc Interv. 2016;9:866-8.

21. Liddon L, Kingerlee R, Barry JA. Gender differences in preferences for psychological treatment, coping strategies, and triggers to help-seeking. BrJClin Psychol. 2018;57(1):42-58.

22. Alipour S, Starovoytov A, Heydari-Kamjani M, Robinson B, Daniele P, Gao M, et al. Frequency and effects of emotional and physical stressors in patients with spontaneous coronary artery dissection. J Am Col Cardiol. 2016;67 (13. Suppl):295.

23. Al-Hussaini A, Adlam D. Spontaneous coronary artery dissection. Heart. 2017;103:1043-51.

24. Plouin P-F, Perdu J, La Batide-Alanore A, Boutouyrie P, Gimenez-RoqueploA-P, JeunemaitreX. Fibromusculardysplasia. OrphanetJ Rare Dis. 2007;2:28.

25. Persu A, Giavarini A, Touze E, et al. European consensus on the diagnosis and management of fibromuscular dysplasia. JHypertens. 2014;32:1367-78.

26. Olin JW, Froehlich J, Gu X, et al. The United States Registry for Fibromuscular Dysplasia: results in the first 447 patients. Circulation. 2012. DOI https://doi.org/10.1161/ CIRCULATIONAHA.112.091223 (ePub ahead of print). 
27. PersuA, Van der Niepen P, TouzéE, etal. Revisiting fibromuscular dysplasia: rationale of the European Fibromuscular Dysplasia Initiative. Hypertension. 2016;68:832-9.

28. Kadian-Dodov D, Gornik HL, Gu X, et al. Dissection and aneurysm in patients with fibromuscular dysplasia: findings from the U.S. Registry for FMD. J Am Coll Cardiol. 2016;68:176-85.

29. Prasad M, Tweet MS, Hayes SN, et al. Prevalence of extracoronary vascular abnormalities and fibromuscular dysplasia in patients with spontaneous coronary artery dissection. Am J Cardiol. 2015;115:1672-7.

30. Liang JJ, Prasad M, Tweet MS, et al. A novel application of CT angiography to detect extracoronary vascular abnormalities in patients with spontaneous coronary artery dissection. JCardiovasc Comput Tomogr. 2014;8:189-97.

31. Toggweiler S, Puck M, Thalhammer C, et al. Associated vascular lesions in patients with spontaneous coronary artery dissection. Swiss Med Wkly. 2012;142:w13538.

32. Johnston N, Schenck-Gustafsson K. Spontaneous coronary artery dissection-A need for raised awareness among healthcare professionals evaluating pregnant and postpartum women with chest pain. Maturitas. 2017;104:123-4.

33. Roth A, Elkayam U. Acute myocardial infarction associated with pregnancy. JAm Coll Cardiol. 2008;52:171-80.

34. Koul AK, Hollander G, Moskovits N, Frankel R, Herrera L, Shani J. Coronary artery dissection during pregnancy and the postpartum period: two case reports and review of literature. Catheter Cardiovasc Interv. 2001;52:88-94.

35. RoyJJ, Klein HZ. Dissecting aneurysm of the coronaryartery. JAMA. 1971;218:1047-8.

36. Bonnet J, Aumailley M, Thomas D, Grosgogeat Y, Broustet JP, Bricaud H. Spontaneous coronary artery dissection: case report and evidence for a defect in collagen metabolism. Eur Heart J. 1986;7:904-9.

37. Wingrove CS, Garr E, Godsland IF, Stevenson JC. 17betaoestradiol enhances release of matrix metalloproteinase2 from human vascular smooth muscle cells. Biochim Biophys Acta. 1998;1406:169-74.

38. Robinowitz M, Virmani R, McAllister HAJ. Spontaneous coronary artery dissection and eosinophilic inflammation: a cause and effect relationship? Am J Med. 1982;72:923-8.

39. Burke AP, Kutys R, Fowler D, Virmani R. Multiple spontaneous coronary artery dissections in association with anomalous origin of right coronary and intramural coronary artery dysplasia. Cardiovasc Pathol. 2004;13:173-5.

40. Bateman AC, Gallagher PJ, Vincenti AC. Sudden death from coronary artery dissection. JClin Pathol. 1995;48:781-4.

41. Tweet MS, Eleid MF, Best PJ, et al. Spontaneous coronary artery dissection: revascularization versus conservative therapy. Circ Cardiovasc Interv. 2014;7:777-86.

42. Alfonso F, Paulo M, Lennie V, et al. Spontaneous coronary artery dissection: long-term follow-up of a large series of patients prospectively managed with a "conservative" therapeutic strategy. Jacc Cardiovasc Interv. 2012;5:1062-70.

43. Tweet MS, Gulati R, Hayes SN. Spontaneous coronary artery dissection. Curr Cardiol Rep. 2016;18:60.

44. Main AE, Starovoytov A, Aymong E, Mancini GBJ, Saw J. Recurrent sponatenous coronayartery dissection. JAm Coll Cardiol. 2016;67(13. Suppl):565.

45. Agrawal V, Kim ESH. Spontaneous coronary artery dissection: cardiac manifestations of vascular disease. Prog Cardiovasc Dis. 2018. https:// doi.org/10.1016/j.pcad.2018. 04.001 (ePub ahead of print). 